\title{
INFLUENCIA DE LA SALINIDAD Y AIREACIÓN EN EL CRECIMIENTO DE Limnobium laevigatum (HUMB. \& BONPL. EX WILLD.) HEINE
}

\section{INFLUENCE OF SALINITY AND AERATION ON THE GROWTH OF Limnobium laevigatum (HUMB. \& BONPL. EX WILLD.) HEINE}

\author{
Adriana Troncoso-Gómez ${ }^{1}$ y Héctor Aponte ${ }^{1,2}$
}

\begin{abstract}
Resumen
Limnobium laevigatum es una planta acuática flotante perteneciente al orden Hydrocharitales, familia Hydrocharitaceae. Esta especie ha demostrado tener un alto valor nutricional, ser eficiente para la fitorremediación y como captadora de carbono. Conocer las mejores condiciones que permitan su propagación es fundamental para plantear su reproducción a gran escala, aprovechando los beneficios que nos brinda. El presente estudio tuvo como objetivo evaluar la influencia de la salinidad y aireación sobre el crecimiento de L. laevigatum en condiciones de laboratorio. Para ello, se diseñó un experimento donde se evaluaron diferentes variables de crecimiento (número de rametos, número de hojas totales, hojas cloróticas, hojas muertas, tamaño de la raíz, peso húmedo total, peso seco total, área foliar y tasa de crecimiento relativo -TCR) a diferentes concentraciones de salinidad (2.2, 2.9 y $3.7 \mathrm{dS} / \mathrm{m}$, consideradas nivel uno, dos y tres, respectivamente) y aireación (con y sin aireación). Los resultados mostraron que los valores más altos de las variables de crecimiento se obtuvieron en los tratamientos sin aireación y con nivel uno de salinidad, obteniendo así la mayor TCR $\left(0.0785 \mathrm{~g} \cdot \mathrm{g}^{-1} \cdot \mathrm{d}^{-1}\right)$. Estos resultados sugieren que, de querer implementar un sistema de propagación a gran escala, no sería necesario incluir sistemas de aireación y trabajar con soluciones nutritivas bajas en sales.
\end{abstract}

Palabras clave: Limnobium laevigatum, estrés osmótico, flujo de aire, Hydrocharitaceae, plantas acuáticas.

\begin{abstract}
Limnobium laevigatum (Hydrocharitales Order, Hydrocharitaceae family) is a floating aquatic plant known to possess a high nutritional value, to be efficient for phytoremediation and as a carbon captor. In order to achieve large scale reproduction and to take advantage of the benefits that this species offers, it is fundamental to recognize best conditions that enable its propagation. The objective of this study was to evaluate the influence of salinity and aeration on the growth of $L$. laevigatum under laboratory conditions. For this, an experiment was designed where different growth variables were evaluated (number of sprigs, total number of leaves, chlorotic leaves, dead leaves, root size, total wet weight, total dry weight, leaf area and relative growth rate -TCR) under different salinity concentrations $(2.2,2.9$ and $3.7 \mathrm{dS} / \mathrm{m}$, considered as level one, two and three, respectively) and aeration conditions (with and without aeration). The results showed that the highest values of the growth variables were obtained in the treatments without aeration and at level one of salinity, obtaining the highest TCR $\left(0.0785 \mathrm{~g} \cdot \mathrm{g}^{-1} \cdot \mathrm{d}^{-1}\right)$. These results suggest that, in order to implement a large-scale propagation system, it would not be necessary to include aeration systems and low salt nutritional solutions can be used.
\end{abstract}

Key words: Limnobium laevigatum, osmotic stress, airflow, Hydrocharitaceae, aquatic plants.

\section{Introducción}

Limnobium laevigatum (Humb. \& Bonpl. ex Willd.)

Heine es una planta acuática flotante perteneciente al orden Hydrocharitales, familia Hydrocharitaceae; el rango de distribución de esta especie abarca Centroamérica y Sudamérica, desde México central y Cuba hasta Buenos Aires en Argentina (Cook \& UrmiKönig, 1983). En el Perú se ha reportado en las regiones de Ucayali, Loreto y en Lima (Brako \& Zarucchi, 1993; Ramirez \& Cano, 2010). Esta especie ha ganado especial interés en los últimos años debido a que presenta un valor nutricional adecuado para ser utilizada como forraje para la acuicultura debido a su valor proteico el cual puede alcanzar un $39.76 \%$ en base seca y bajo contenido de fibra $(7.63 \%)$ el cual se encuentra dentro del rango recomendado en dietas para peces (Fleming et al., 1996; Corti \& Schlatter, 2002; Aponte et al., 2013). También se ha demostrado que es eficiente en la fitorremediación de aguas residuales industriales (Valderrama, 1996; Martínez, 2014) y que 
Enero - Julio 2020

a su vez puede ser utilizada con fines de captura de carbono si se lograse su reproducción a gran escala (Aponte, 2017).

Se han realizado estudios sobre las condiciones de cultivo para L. laevigatum, donde se ha encontrado que se obtiene mayor crecimiento en soluciones nutritivas con 68 ppm de nitratos y 62 ppm de fosfatos, y con la mayor cantidad de luz ambiental disponible en laboratorio $\left(181.6 \mu \mathrm{mol} \cdot \mathrm{m}^{-2} \cdot \mathrm{s}^{-1}\right)$, logrando una capacidad de carga de $0.178 \mathrm{~g} / \mathrm{cm}^{2}$ (Aponte \& Pacherres, 2013; Aponte, 2016). A pesar de ello, son necesarios mayores estudios a fin de optimizar su crecimiento en laboratorio para poder aprovechar sus cualidades antes descritas.

La salinidad y la aireación juegan un rol importante en el crecimiento de las plantas. La presencia de aireación beneficia el crecimiento de las hojas y el buen funcionamiento radicular ya que favorece la respiración aerobia en las raíces y evita la acumulación de materiales o microorganismos que pueden ser dañinos (Chérif et al., 1997; Domínguez, 2003). El sodio puede actuar como sustituto parcial del potasio y participa en la apertura y cierre de estomas, lo cual ayuda a regular el equilibrio interno de agua; el cloro interviene en la osmosis y en el equilibrio iónico en el interior de las células; la deficiencia de estos dos minerales puede producir marchitamiento y reducción en el crecimiento foliar y de la raíz; por otro lado, el exceso de sodio y cloro puede inhibir el crecimiento, ya que causa estrés osmótico y, además, interfiere en la captación de otros nutrientes esenciales como el calcio y potasio, generando desequilibrios nutricionales (Haller et al., 1974; Wendeou et al., 2013; Beltrano \& Gimenez, 2015).

La especie en estudio habita comúnmente en ambientes lénticos de agua dulce y salobre, reportándose en ambientes con conductividades de hasta $3.38 \mathrm{dS} / \mathrm{m}$ (Brako \& Zarucchi, 1993; Ramirez \& Cano, 2010; Howard et al., 2016; Aponte, 2017), pudiendo cambiar su rendimiento según las condiciones del medio. En este contexto, el presente estudio tiene como finalidad evaluar la influencia de la salinidad y aireación sobre el crecimiento de $L$. laevigatum para contribuir al desarrollo su cultivo en laboratorio.

\section{Metodología \\ Colecta}

Los rametos de L. laevigatum fueron colectados de la zona de amortiguamiento del Refugio de Vida Silvestre Pantanos de Villa (RVSPV) teniendo la autorización del Serivicio forestal y de fauna silvestre ( ${ }^{\circ}$ AUT-IFL-2017-031). La zona de toma de muestra corresponde a uno de los canales que alimenta el pantano (18L 284263.97 E - 8649114.35 S, 5 msnm). Las plantas fueron llevadas al laboratorio de Investigación de Biología Marina de la Universidad Científica del Sur (Lima, Perú) en donde se realizó la aclimatación y experimentación en condiciones de laboratorio.

\section{Aclimatación}

Los rametos colectados se limpiaron y propagaron en tanques de 20 1, con la solución nutritiva sugerida para su propagación óptima (Aponte \& Pacherres, 2013) (Tabla 1). Esta etapa tuvo una duración de tres meses, de junio a agosto. Diariamente fueron medidos los parámetros de temperatura $\left({ }^{\circ} \mathrm{C}\right)$ y humedad $(\%)$, iluminación $\left(\mu \mathrm{mol} \cdot \mathrm{m}^{-2} \cdot \mathrm{s}^{-1}\right)$ y fotoperiodo $\left(\mathrm{h} \cdot \mathrm{d}^{-1}\right)($ Tabla 2).

Tabla 1. Concentración de elementos en partes por millón (ppm) de la solución utilizada basándose en Aponte \& Pacherres (2013).

\begin{tabular}{lr}
\hline Elemento & {$[\mathrm{ppm}]$} \\
\hline $\mathrm{N}$ & 68.08 \\
$\mathrm{P}$ & 62.90 \\
$\mathrm{~K}$ & 17.02 \\
$\mathrm{Mg}$ & 27.47 \\
$\mathrm{~S}$ & 54.22 \\
$\mathrm{Ca}$ & 116.20 \\
$\mathrm{Fe}$ & 0.39 \\
$\mathrm{Mn}$ & 0.13 \\
$\mathrm{Zn}$ & 0.14 \\
$\mathrm{Cu}$ & 0.04 \\
$\mathrm{~B}$ & 0.77 \\
$\mathrm{Mo}$ & 0.02 \\
\hline
\end{tabular}

\section{Diseño experimental}

Se trabajó con seis tratamientos, con quince repeticiones por cada tratamiento, en los cuales se utilizaron rametos de dos hojas producidos durante la etapa de aclimatación. Cada repetición consistió en un rameto ubicado en un vaso de precipitado de $600 \mathrm{ml}$, el cual contenía $300 \mathrm{ml}$ de solución nutritiva (según las condiciones descritas por Aponte \& Pacherres, 2013;

Tabla 2. Parámetros del laboratorio durante el periodo de aclimatación y experimentación.

\begin{tabular}{lcc}
\hline \multirow{2}{*}{ Parámetro } & \multicolumn{2}{c}{ Promedio \pm Desviación estándar } \\
\cline { 2 - 3 } & Aclimatación & Experimentación \\
\hline Temperatura ambiente $\left({ }^{\circ} \mathrm{C}\right)$ & $22.3 \pm 1.1$ & $22.3 \pm 1.6$ \\
Temperatura del agua $\left({ }^{\circ} \mathrm{C}\right)$ & $22 \pm 1.4$ & $21.8 \pm 1.5$ \\
Luz $\left(\mu \mathrm{mol} \cdot \mathrm{m}^{-2} \cdot \mathrm{s}^{-1}\right)$ & $95.8 \pm 150.1$ & $106.8 \pm 80.9$ \\
Fotoperiodo $\left(\mathrm{h} \cdot \mathrm{d}^{-1}\right)$ & $11.4 \pm 0.01$ & $12.8 \pm 0.01$ \\
Humedad $(\%)$ & $65.0 \pm 10.7$ & $65 \pm 9.4$ \\
\hline
\end{tabular}


Aponte, 2017) (Tabla 3). Los envases fueron distribuidos siguiendo un orden aleatorio y cubiertos lateralmente por un plástico de color negro para reducir la exposición de la solución a la luz.

Tabla 3. Promedio \pm Desviación estándar de $\mathrm{pH}$ y conductividad por tratamiento a lo largo del experimento. Se muestra si el tratamiento tiene o no aireación.

\begin{tabular}{|c|c|c|c|}
\hline \multirow[b]{2}{*}{ 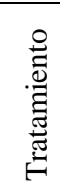 } & \multicolumn{3}{|c|}{ Promedio \pm Desviación estándar } \\
\hline & $\mathrm{pH}$ & Conductividad $(\mathrm{dS} / \mathrm{m})$ & 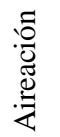 \\
\hline $\mathrm{t} 1$ & $8.59 \pm 0.11$ & $2.29 \pm 0.23$ & $\mathrm{Si}$ \\
\hline $\mathrm{t} 2$ & $8.52 \pm 0.11$ & $2.27 \pm 0.23$ & No \\
\hline $\mathrm{t} 3$ & $8.68 \pm 0.17$ & $2.95 \pm 0.18$ & $\mathrm{Si}$ \\
\hline $\mathrm{t} 4$ & $8.55 \pm 0.18$ & $2.97 \pm 0.19$ & No \\
\hline t5 & $8.66 \pm 0.17$ & $3.72 \pm 0.20$ & $\mathrm{Si}$ \\
\hline t6 & $8.51 \pm 0.16$ & $3.72 \pm 0.19$ & No \\
\hline
\end{tabular}

Se evaluaron tres niveles de salinidad $(2.2,2.9$ y 3.7 $\mathrm{dS} / \mathrm{m}$, nivel uno ( $\mathrm{t} 1 \mathrm{y} \mathrm{t} 2)$, nivel dos ( $\mathrm{t} 3 \mathrm{y} \mathrm{t} 4)$ y nivel tres (t5 y t6) respectivamente) y dos niveles de aireación (con y sin aireación). Las diferentes concentraciones salinas se consiguieron mediante la adición de $\mathrm{NaCl}$ en estado sólido a una solución madre con agua potable hasta alcanzar la salinidad deseada. La conductividad (dS/m) y el pH para cada tratamiento se verificaron antes y después de cada recambio de agua con un conductímetro Hanna modelo HI8633 y un pHmetro Crison $\mathrm{pH} 25+$.

El método de aireación utilizado fue el sugerido en Beltrano \& Gimenez (2015) y consistió en un sistema de burbujeo con una bomba de aire de $1.5 \mathrm{hp} \mathrm{y}$ mangueras. Para garantizar la homogeneidad de aireación en todas las réplicas, se reguló el flujo de aire de cada manguera con válvulas plásticas; éste se mantuvo en $1.73 \cdot 10^{-6} \pm 0.219 \cdot 10^{-6} \mathrm{~m}^{3} \cdot \mathrm{s}^{-1}$. Cada siete días se tomaron mediciones de las siguientes variables de crecimiento: número de rametos, número de hojas totales, hojas cloróticas (\%), hojas muertas (\%), tamaño de la raíz $(\mathrm{cm})$, peso húmedo total $(\mathrm{g})$, y al finalizar el ensayo se midió el peso seco total $(\mathrm{g})$, área foliar $\left(\mathrm{cm}^{2}\right)$ y se calculó la tasa de crecimiento relativo (TCR), la cual es la ganancia de biomasa en un tiempo determinado en relación a la biomasa inicial $\left(\mathrm{g} \cdot \mathrm{g}^{-1} \cdot \mathrm{d}^{-1}\right)$ definido como $r$ en la Ecuación 1:

$$
r=\frac{\overline{\operatorname{LnWF}_{\mathrm{i}}}-\overline{\mathrm{LnWI}_{\mathrm{i}}}}{\mathrm{t}_{2}-\mathrm{t}_{1}} \ldots \text { Ecuación } 1 .
$$

Donde WF y WI son los pesos húmedos (g) final e inicial de cada replica (i) dentro de cada tratamiento, y el tiempo $\left(\mathrm{t}_{2}-\mathrm{t}_{1}\right)$ fue el número de días (d) del experimento (Hoffmann \& Poorter, 2002); esta variable ha sido utilizada para estimar la TCR en estudios previos (Al-Nozaily, 2001; Wendeou et al., 2013;
Aponte \& Pacherres, 2013). Los pesos (g) se registraron utilizando una balanza electrónica Digiweigh (Modelo DW-100AS; $0.01 \mathrm{~g}$ de precisión).

Con la finalidad de mantener las condiciones a lo largo del experimento, se removió la materia muerta de los envases de vidrio y la solución de cada envase fue cambiada por una nueva a la misma concentración dos veces por semana, esto a fin de mantener las mismas condiciones de nutrientes a lo largo del experimento.

El experimento finalizó cuando uno de los tratamientos llegó a ocupar la totalidad de la superficie del agua lo cual ocurrió a los 35 días, de esta manera se evitó que el efecto de la densidad interfiriera en los resultados.

Análisis de datos

Se utilizó un diseño completo al azar con arreglo factorial, donde los factores fueron 1)

salinidad y, 2) aireación. Se verificó el efecto individual y combinado de las variables salinidad y aireación en el crecimiento de L. laevigatum. Dado que los datos no cumplían con los parámetros necesarios para aplicar pruebas paramétricas $(\mathrm{p}<0.05$ para el test de Shapiro Wilk y para la prueba de homocedasticidad de Levene), se utilizó la prueba no paramétrica de Kruskal Wallis y la prueba a posteriori de Mann Whitney. Se realizó un NPMANOVA de doble vía para visualizar el efecto de la interacción de ambas variables (salinidad y aireación) sobre los parámetros de crecimiento. Las pruebas y los gráficos fueron realizados utilizando el programa PAST (Hammer et al.,2001), y Excel 2016 (C Microsoft bajo licencia) y la aplicación BoxplotR (Spitzer et al., 2014).

\section{Resultados}

Se encontró diferencias significativas $(p<0.05)$ entre los tratamientos sin aireación (t2 y t4) y los tratamientos con aireación $(\mathrm{t} 1, \mathrm{t} 3, \mathrm{t} 5)$ para el peso fresco, número de hojas, tasa de crecimiento, área foliar y peso seco; en todos estos casos los promedios de los tratamientos sin aireación fueron más elevados. El tratamiento t5 fue el que obtuvo promedios más bajos en todos los parámetros de crecimiento, a excepción del porcentaje de hojas cloróticas y muertas (Figura 1).

En la Figura 1 se puede observar que el tratamiento t5 presentó mayor porcentaje de hojas cloróticas. Los tratamientos con menor salinidad ( $\mathrm{t} 1 \mathrm{y} \mathrm{t}$ 2) fueron los que presentaron menor porcentaje de hojas cloróticas, siendo el t 2 el único que se diferenció estadísticamente del resto $(p<0.05, p=0.00024)$. Para la mortalidad, no se observaron diferencias significativas $(\mathrm{p}>0.05, \mathrm{p}=$ 0.529); no obstante, los tratamientos con mayor salinidad ( 15 y t6) fueron los que presentaron un mayor porcentaje de hojas muertas.

La TCR fue mayor en los tratamientos sin aireación con una media de $0.0785 \mathrm{~g} \cdot \mathrm{g}^{-1} \cdot \mathrm{d}^{-1}, 0.0783 \mathrm{~g} \cdot \mathrm{g}^{-1} \cdot \mathrm{d}^{-1} \mathrm{y}$ $0.0726 \mathrm{~g} \cdot \mathrm{g}^{-1} \cdot \mathrm{d}^{-1}$ para los tratamientos $\mathrm{t} 2$, t4 y $\mathrm{t} 6$, respectivamente, en comparación con los tratamientos con aireación (Figura 2). El área foliar también fue 
mayor en los tratamientos sin aireación con una media de $28.3,27.9$ y $20.4 \mathrm{~cm}^{2}$ para los tratamientos $\mathrm{t} 2, \mathrm{t} 4 \mathrm{y}$ t6, respectivamente; los tratamientos con aireación t1, t3 y t5 tuvieron un área foliar de $19.2,17$ y $12.1 \mathrm{~cm}^{2}$, respectivamente (Figura 3). Se obtuvo un mayor peso seco en los tratamientos sin aireación con una media de $0.10,0.11$ y 0.09 g para los tratamientos $\mathrm{t} 2$, $\mathrm{t} 4 \mathrm{y} \mathrm{t} 6$, respectivamente; los tratamientos con aireación $\mathrm{t} 1, \mathrm{t} 3 \mathrm{y}$ t5 tuvieron un peso seco promedio de $0.07,0.05$ y 0.04 $\mathrm{g}$, respectivamente (Figura 4 ).

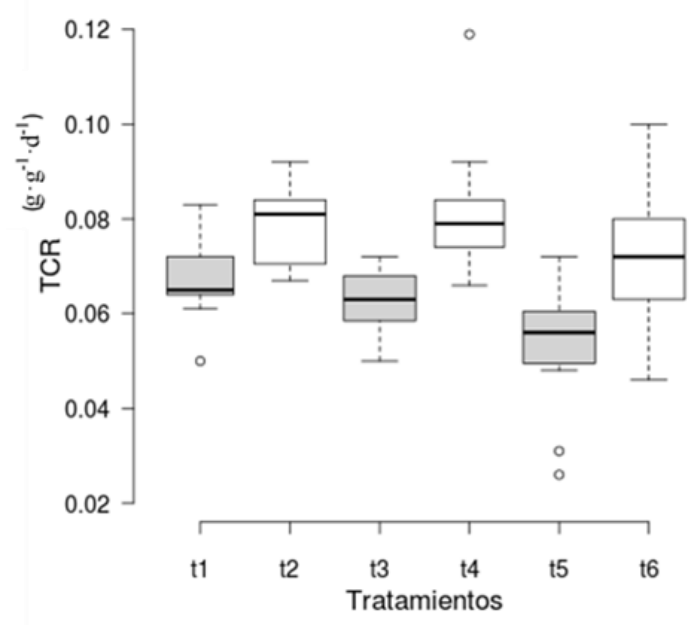

Figura 2. Tasa de crecimiento relativo (TCR) de Limnobium laevigatum por tratamiento. Tratamientos sin aireación en blanco y con aireación en gris.

En la prueba NPMANOVA de doble vía se observó que no existe una interacción de ambas variables ( $\mathrm{p}=$ $0.8687 ; \mathrm{F}=-1.45)$. Sin embargo, se observó que hay un efecto significativo de la salinidad y la aireación por separado $(\mathrm{p}=0.0014, \mathrm{p}=0.0001 ; \mathrm{F}=6.01$ y $\mathrm{F}=28.72$ para salinidad y de la aireación, respectivamente), sobre el crecimiento de L. laevigatum.

\section{Discusión}

Los resultados obtenidos indican que a mayor salinidad esta especie presenta un menor rendimiento. Esto se puede deber a que la salinidad disminuye el potencial hídrico del medio, restringiendo así la absorción de agua generando un estrés osmótico. También se podría estar generando un desequilibrio nutricional ya que el aumento de cloro y sodio produce toxicidad y genera una competencia directa en la absorción de otros iones esenciales para el crecimiento y metabolismo de la planta como el $\mathrm{K}$; el exceso de sodio inhibe el funcionamiento enzimático (Dell'Amico \& Parra, 2005; Parida \& Das, 2006; Alcaraz, 2012; Beltrano \& Gimenez, 2015). Estos resultados coinciden con el estudio de Haller et al. (1974) sobre el efecto de la salinidad en plantas acuáticas, donde se mostró que las concentraciones de sal de 1660 ppm, y 2500 ppm, afectaron el crecimiento de Pistia stratiotes L. y Eichornia crassipes (Mart.)
Solms, respectivamente. Por otro lado, el mismo estudio menciona que el crecimiento de Lemna minor L. se vio favorecido con las concentraciones de sal de 830 ppm, 1660 ppm, 2500 ppm y 3330 ppm, siendo considerada esta especie como una de las más tolerantes a la salinidad; las otras especies (Hydrilla verticillata Royle, Myriopyllum spicatum L., Najas quadalupensis (Spreng.) Magnus, Vallisneria americana Michx., Azolla caroliniana Willd., y Salvinia rotundifolia Willd.) tuvieron un declive en el crecimiento a medida que la concentración de salinidad se incrementó. Especies como Spirodela polyrrhiza (L.) Schleid, se ven afectadas también por la salinidad, inhibiendo su crecimiento a conductividades mayores a $2 \mathrm{dS} / \mathrm{m}$ (Wendeou et al., 2013), concordando esta reacción con la encontrada en el presente trabajo para L. laevigatum.

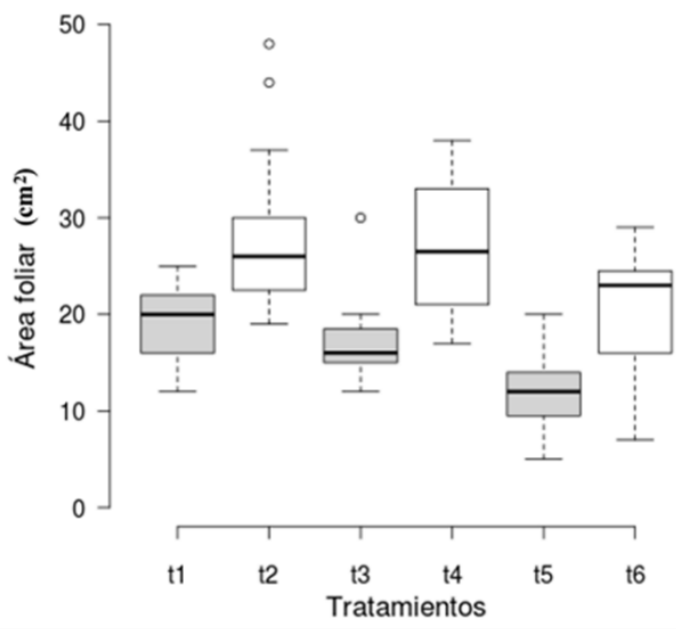

Figura 3. Área foliar de Limnobium laevigatum por tratamiento. Tratamientos sin aireación en blanco y con aireación en gris.

L. laevigatum mostró un mejor crecimiento en los tratamientos sin aireación lo que sugiere que esta planta no requiere de dicho sistema para desarrollarse. Esto se puede deber a que la zona que habita la planta presenta baja o nula recirculación, siendo eficiente en el uso de oxígeno. Esto ocurre con plantas acuáticas que pueden crecer en condiciones hipóxicas debido a su capacidad de transportar oxigeno de las hojas a las raíces (Greenwood \& Goodman, 1971) o por la presencia de aerénquimas, lo cual se ha demostrado en otras plantas acuáticas como Eichhornia crassipes, que facilita el transporte de gases como el oxígeno, estas estructuras tienden a desarrollarse en respuesta a condiciones bajas de oxígeno (kawase \& Whitmoyer, 1980; Rubio et al., 2015). La tolerancia a bajas concentraciones de oxígeno también se ha reportado en la planta acuática Nasturtium officinale Aiton ( Fernández, 2013). 


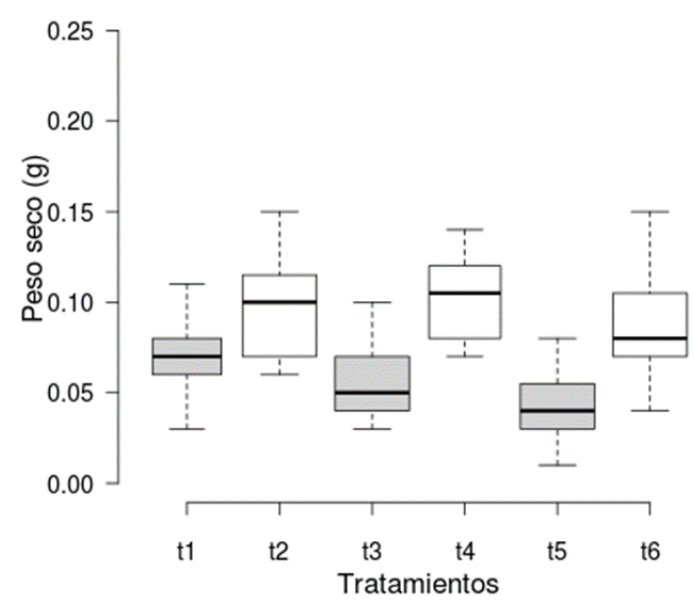

Figura 4. Peso seco total de Limnobium laevigatum por tratamiento. Tratamientos sin aireación en blanco y con aireación en gris.

Una de las limitaciones del presente estudio correspondió a la iluminación, la cual estuvo alrededor de $106.8 \pm 80.9\left(\mu \mathrm{mol} \cdot \mathrm{m}^{-2} \cdot \mathrm{s}^{-1}\right)$, siendo este valor menor a lo sugerido para lograr el crecimiento más óptimo de esta especie $\left(181.6 \mu \mathrm{mol} \cdot \mathrm{m}^{-2} \cdot \mathrm{s}^{-1}\right)$ (Aponte, 2016); ello no afecta la interpretación de los datos y explica las diferencias en las TCR encontrados en el presente trabajo (la cual fue menor que la de dicho estudio previo, muy probablemente por esa diferencia lumínica).

El presente estudio indica que, por separado, las variables de salinidad y aireación tienen un efecto sobre el crecimiento; sin embargo, no existe un efecto combinado entre estas variables, lo cual también se puede apreciar en la Figura 2, encontrándose tratamientos con un rendimiento bastante similar a pesar de tener diferente salinidad (por ejemplo, el t2 y t6). Esto sugiere que en ausencia de salinidad el crecimiento es más homogéneo, evitando los daños propios del exceso de dicho recurso.

El presente trabajo permite tener una idea de las condiciones que requiere esta especie para su propagación adecuada. En este caso, se muestra que la aireación es innecesaria, lo que optimizaría considerablemente el presupuesto de implementación de un sistema para su propagación a gran escala. Asimismo, se reafirman los estudios previos en donde no es necesaria la adición de sales al medio.

\section{Conclusiones}

Limnobium laevigatum presentó un mejor crecimiento con el nivel uno de salinidad (1454 \pm 145 ppm) y en sistemas sin aireación. No se comprobó un efecto combinado entre la salinidad y la aireación sobre el desarrollo de esta especie.

\section{Agradecimientos}

Agradecemos el apoyo logístico de la Facultad de Ciencias Veterinarias y Biológicas (carrera de Biología Marina, Universidad Científica del Sur). Asimismo, agradecemos a la Dra. Joyce del Pino, al Blgo. Aldo Indacochea y al Mg. Juan Carlos Francia por su apoyo en la logística del experimento. Finalmente, agradecemos a Angela Acosta, Angélica Aguirre, Edgar López, Erin Villanueva, Rebeca Campos y Frank Huanca quienes apoyaron en el desarrollo del experimento.

\section{Literatura citada}

Alcaraz F. 2012. Salinidad y vegetación. En: Geobotánica, Tema 18. Universidad de Murcia. Disponible en: https://www.um.es/docencia/geobotanica/ficheros/tema1 8.pdf.

Al-Nozaily F.A. 2001. Performance and process analysis of duckweed-covered sewage lagoons for high strength sewage. Disertación Doctoral. Delft University of Technology International Institute of Hydraulic and Environmental Engineering. Delft-Holland.

Aponte H. \& Pacherres C.O. 2013. Crecimiento y propagación de Limnobium laevigatum (Hydrocharitaceae) bajo diferentes concentraciones de nutrientes. The Biologist, 11(1): 69-78. Lima, Perú. Consultable en http://sisbib.unmsm.edu.pe/bvrevistas/biologist/v11_n1/ pdf/a6v11n1.pdf al 04 de mayo de 2019.

Aponte H. 2016. Crecimiento de Limnobium laevigatum (Hydrocharitaceae) Bajo Diferentes Condiciones Lumínicas. The Biologist, 14(2): 297-305. Consultable en http://aplicaciones.cientifica.edu.pe/repositorio/catalogo _0/134.pdf al 04 de mayo de 2019.

Aponte H. 2017. Productividad de Limnobium laevigatum (Hydrocharitaceae) Bajo Condiciones de Laboratorio. Polibotánica, 0(44): 157-166. DOI: 10.18387/polibotanica.44.12.

Aponte H., Segura C. \& Francia J.C. 2013. Análisis Químico Proximal de Limnobium laevigatum y su Potencial Para $\mathrm{Su}$ Uso Como Forraje. Cientifica, 10(2): 158-67. Consultable $\mathrm{http} / / /$ aplicaciones.cientifica.edu.pe/repositorio/catalogo/ _data/6.pdf al 03 de mayo de 2019.

Beltrano J. \& Gimenez D. 2015. Cultivo en hidroponía. 1a ed., Universidad Nacional de La Plata. La Plata.

Boettcher C.T. 2007. Variación comparativa de biomasa estacional en dos macrófitas de la Región de Valdivia, Chile. Tesis para licenciatura en Ciencias Biológicas. Universidad Austral de Chile. En http://cybertesis.uach.cl/tesis/uach/2007/fcb673v/doc/fcb 673v.pdf al 04 de mayo de 2019.

Brako L. \& Zarucchi J.L. 1993. Catálogo de las angiospermas y gimnospermas del Perú. 45th ed. Missouri Botanical Garden. St. Louis, Missouri.

Chérif M., Tirilly Y. \& Bélanger R.R. 1997. Effect of oxygen concentration on plant growth, lipidperoxidation, and receptivity of tomato roots to Pythium F under hydroponic conditions. European Journal of Plant Pathology, 103(3): 255-264. DOI: https://doi.org/10.1023/A:1008691226213. 
Cook C. \& Urmi-König K. 1983. A revision of the genus Limnobium including Hydromystria (Hydrocharitaceae). Aquatic Botany, 17(1): 1-27. DOI: https://doi.org/10.1016/0304-3770(83)90015-3.

Corti P. \& Schlatter R. 2002. Feeding ecology of the blacknecked swan Cygnus melancoryphus in two wetlands of southern Chile. Studies on Neotropical Fauna and Environment, 37(1): 9-14. DOI https://doi.org/10.1076/snfe.37.1.9.2118.

Dell'Amico J.M. \& Parra M. 2005. Efecto del estrés por $\mathrm{NaCl}$ en el contenido de cloruros, el potencial osmótico real y el crecimiento de dos cultivares de tomate cubanos. Cultivos Tropicales, 26(2): 39-44. Disponible en http://www.redalyc.org/articulo.oa?id=193215934007 al 04 de mayo de 2019.

Domínguez A. 2003. Transporte de oxígeno a través de plantas acuáticas. Scientia Et Technica, 1(21): 159-62. DOI: http://dx.doi.org/10.22517/23447214.7529.

Fernández M. 2013. Efecto de diferentes niveles de aireación de la solución nutritiva sobre el crecimiento y la calidad de canónigos y berros cultivados en bandejas flotantes. ETSIA Cartagena. Universidad Politécnica de Cartagena. URI: http://hdl.handle.net/10317/3130.

Fleming A., Van Bameveld R. \& Hone P.W. 1996. The Development of Artificial Diets for Abalone: A Review and Future Directions. Aquaculture, 140(3): 5-53. DOI: 10.1016/0044-8486(95)01184-6.

Greenwood D. \& Goodman D. 1971. Studies on the supply of oxygen to the roots of mustard seedlings (Sinapis alba L.). New phytol, 70(1): 85-96. DOI: https://doi.org/10.1111/j.1469-8137.1971.tb02513.x.

Haller W.T., Sutton D.L. \& Barlowe W.C. 1974. Effects of salinity on growth of several aquatic macrophytes. Ecology, 55(4): 891-894. DOI: https://doi.org/10.2307/1934427.

Hammer Ø., Harper D.A.T. \& Rayan P.D. 2001. PAST: Paleontological statistics software package for education and data analysis. Palaeontologia Electronica, 4(1): 1-9. Consultable en https://palaeoelectronica.org/2001_1/past/past.pdf al 04 de mayo de 2019.
Hoffmann W.A. \& Poorter H. 2002. Avoiding bias in calculations of relative growth rate. Annals of Botany, 90(1): 37-42. DOI: 10.1093/aob/mcf140.

Howard G., Hyde M. \& Bingham M. 2016. Alien Limnobium laevigatum (Humb. \& Bonpl. Ex Willd.) Heine (Hydrocharitaceae) becoming prevalent in Zimbabwe and Zambia. BioInvasions Records, 5(4): 221225. DOI: 10.3391/bir.2016.5.4.05. https://www.reabic.net/journals/bir/2016/4/BIR_2016_H oward_etal.pdf al 04 de mayo de 2019.

Kawase M. \& Whitmoyer E. R. 1980. Aerenchyma development in waterlogged plants. American Journal of Botany, 67(1): 18-22.

Martínez N. 2014. Evaluación de La Capacidad de Limnobium laevigatum Para Fitorremediar Agua Residual de La Industria Textil. Tesis de Licenciatura. Universidad Veracruzana.

Parida A.K. \& Das A.B. 2006. Salt tolerance and salinity effects on plants: a review. Ecotoxicology and Environmental Safety, 60(3): 324-339. DOI: 10.1016/j.ecoenv.2004.06.010.

Ramirez D.W. \& Cano A. 2010. Estado de La Diversidad de La Flora Vascular de Los Pantanos de Villa (Lima - Perú). Revista Peruana de Biología, 17(1): 111-114. DOI: http://dx.doi.org/10.15381/rpb.v17i1.58.

Rubio A.V., Valerio G.A. \& Ferrufino L. 2015. Anatomía caulinar $y$ foliar de tres especies de plantas acuáticas. Portal de la Ciencia, 8: 31-44.

Spitzer M., Wildenhain J., Rappsilber J. \& Tyers M. 2014. BoxPlotR: a web-tool for generation of box plots. Nature Methods, 11(2): 121-122. DOI: 10.1038/nmeth.2811.

Valderrama L.T. 1996. Uso de Dos Especies de Macrófitas Acuáticas, Limnobium laevigatum Y Eichhornia crassipes Para El Tratamiento de Aguas Residuales Agro Industriales. Universitas scientiarum, 3(1): 83-97. Consultable en https://revistas.javeriana.edu.co/index.php/scientarium/a rticle/view/5058 al 03 de mayo de 2019.

Wendeou S., Aina M., Crapper M., Adjovi E. \& Mama D. 2013. Influence of salinity on duckweed growth and duckweed based wastewater treatment system. Journal of Water Resources and Protection,No 5(10): 993-999. DOI: https://doi.org/10.4236/jwarp.2013.510103. 


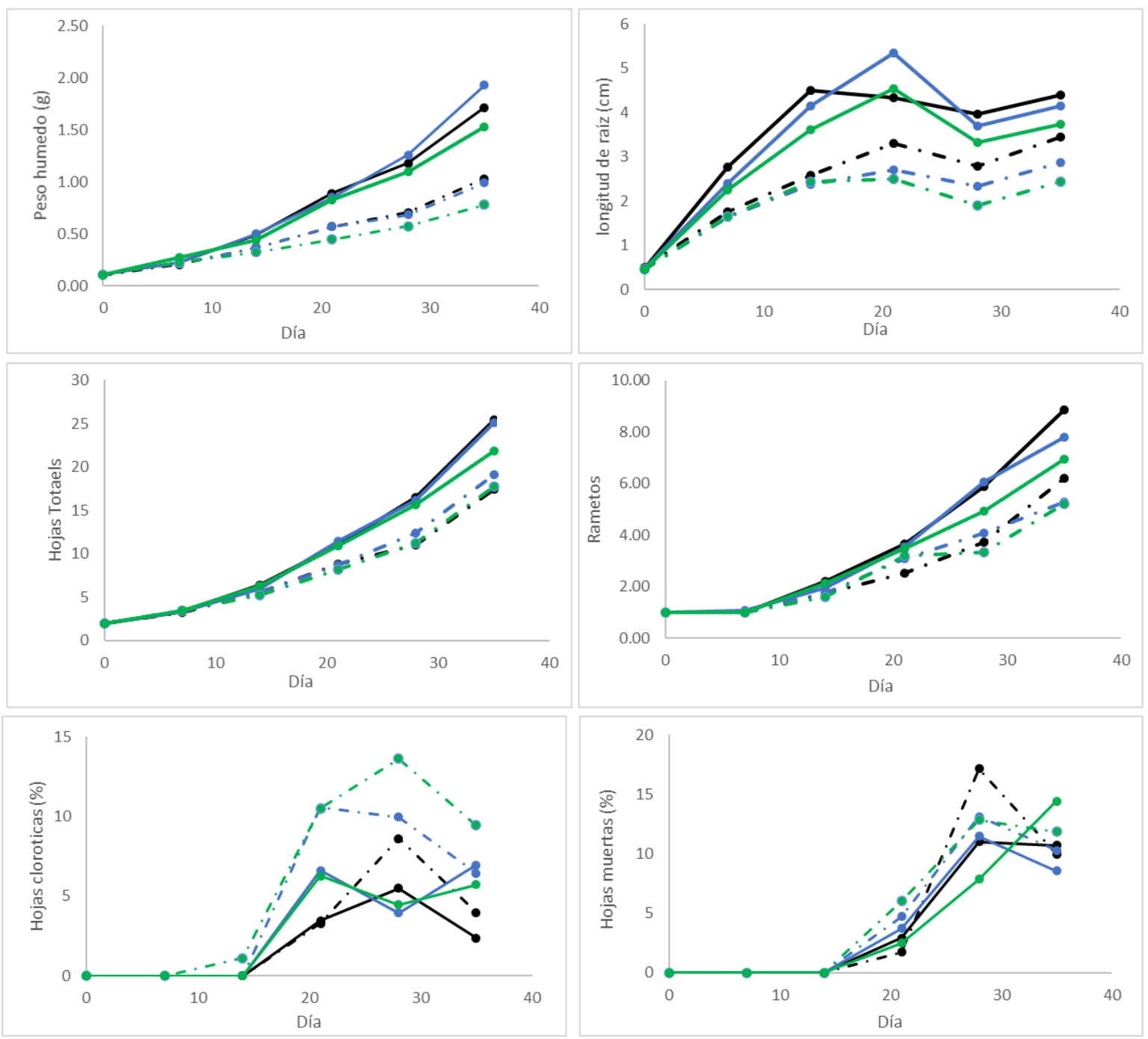

Figura 1. Medias de A: peso húmedo, B: longitud de Raíz, C: número de hojas, D: número de rametos, E: porcentaje de hojas muertas y F: porcentaje de hojas cloróticas, de Limnobium laevigatum durante los 35 días del experimento para cada tratamiento. Las líneas continuas y entre cortadas representan los tratamientos sin y con aireación respectivamente. Salinidad baja ( $\square)$; media $(\square)$ y alta ( $\square)$.

${ }^{1}$ Carrera de Biología Marina / Universidad Científica del Sur. Av. Antigua Carretera Panamericana Sur, km 19 / Villa El Salvador / Lima 42 / Perú. adriana_troncosogomez@ hotmail.com.

${ }^{2}$ Laboratorio de Florística / Museo de Historia Natural / UNMSM. Av. Arenales 1256, Jesús María / Lima 11 / Perú. haponte@cientifica.edu.pe. 